\title{
El trabajo práctico de laboratorio en clase de ciencias naturales durante la pandemia: experiencias en Argentina y Colombia
}

Recepción: 18/06/2021 | Revisión: 19/09/2021 | Aceptación: 31/01/2022 | Publicación: 01/03/2022

\author{
Fanny ANGULO-DELGADO \\ Universidad de Antioquia \\ fanny.angulo@udea.edu.co \\ https://orcid.org/oooo-0003-4458-598X
}

Alejandro CALLE-RESTREPO

Universidad de Antioquia

alejandro.caller@udea.edu.co

https://orcid.org/oooo-0001-9007-1977

(D) Carlos SOTO-LOMBANA

Universidad de Antioquia

carlos.soto@udea.edu.co

https://orcid.org/oooo-ooo3-1475-3153
Las didácticas específicas en tiempos de COVID-19

\author{
Erica ZORRILLA \\ Universidad Nacional de San Juan \\ ericagabriela@gmail.com \\ https://orcid.org/oooo-0oo1-6460-3319
}

D Claudia Alejandra MAZZITELLI
Universidad Nacional de San Juan
claudiamazz@hotmail.com
https://orcid.org/o0o0-0002-1199-4843

Resumen: La enseñanza de las Ciencias Naturales, atendiendo al carácter experimental de estas disciplinas, incorpora como una estrategia didáctica fundamental los trabajos prácticos de laboratorio (TPL). Por esto, cuando se estableció indefinidamente la educación remota de emergencia en el marco de la pandemia, surgió la pregunta sobre qué decisiones estaban tomando los profesores de Ciencias Naturales respecto a los TPL. En este artículo se presentan las respuestas de 35 profesores colombianos y 51 profesores argentinos a una encuesta realizada a fin de conocer sus opiniones y acciones sobre la realización de TPL en este contexto. Los resultados muestran que los profesores adaptaron sus estrategias de enseñanza, apoyándose en los recursos digitales disponibles y replanteando los objetivos, a fin de realizar actividades experimentales. No obstante, también se identificaron prácticas que son irremplazables en su modalidad presencial, según las metas curriculares previstas, especialmente en educación superior. Los docentes evidenciaron ser muy sensibles a las implicaciones de las condiciones socioeconómicas de sus estudiantes, particularmente en contextos rurales donde no hay acceso a internet, de manera que ellos asumieron los costos de ciertas prácticas. Queda por seguir estudiando el impacto en la educación en ciencias de esta modalidad de los TPL durante la pandemia.

Palabras clave: trabajos prácticos; prácticas de laboratorio; ciencias naturales; pandemia. 


\title{
PRACTICAL LABORATORY WORK IN NATURAL SCIENCES CLASS DURING THE PANDEMIC: EXPERIENCES IN ARGENTINA AND COLOMBIA
}

\begin{abstract}
Teaching Natural Sciences, considering the experimental nature of these disciplines, incorporates practical lab work (PLW) as a fundamental didactic strategy. For this reason, when emergency remote education was established indefinitely in the context of the pandemic, the question arose as to what decisions the natural sciences teachers were making regarding the PLW. This article presents the responses of 35 Colombian professors and 51 Argentine professors to a survey carried out to find out their opinions and actions on carrying out PLW in this context. The results show that the teachers adapted their teaching strategies, relying on available digital resources and rethinking the objectives, in order to carry out experimental activities. However, they also identified some practices that are irreplaceable in their face-to-face mode, regarding the planned curricular goals, especially in higher education. Teachers proved to be very sensitive to the implications of their students' socioeconomic conditions, particularly in rural contexts where there is no internet access. Consequently, the teachers themselves assumed the cost of certain practices. The impact on science education of this PLW modality during the pandemic remains to be further explored.
\end{abstract}

Keywords: practical work; lab; natural sciences; pandemic.

\section{EL TREBALL PRÀCTIC DE LABORATORI A CLASSE DE CIÈNCIES NATURALS DURANT LA PANDEMIA: EXPERIÈNCIES A ARGENTINA I COLÒMBIA}

Resum: L'ensenyament de les Ciències Naturals, tenint en compte el caràcter experimental d'aquestes disciplines, incorpora com a estratègia didàctica fonamental els treballs pràctics de laboratori (TPL). Per aquest motiu, quan es va establir indefinidament l'educació remota d'emergència en el marc de la pandèmia, va sorgir la pregunta sobre quines decisions estaven prenent els professors de Ciències Naturals respecte del TPL. En aquest treball es presenten les respostes de 35 professors colombians i 51 d'argentins a una enquesta realitzada per conèixer les seves opinions i accions sobre la realització de TPL en aquest context. Els resultats mostren que els professors van adaptar les seves estratègies d'ensenyament, fent ús dels recursos digitals disponibles i replantejant els objectius, per tal de realitzar activitats experimentals. No obstant això, també es van identificar pràctiques que són insubstituïbles en la seva modalitat presencial, segons els objectius curriculars previstos, especialment a l'educació superior. Els docents van evidenciar ser molt sensibles a les implicacions de les condicions socioeconòmiques dels seus estudiants, especialment en contextos rurals on no hi ha accés a internet. En conseqüència, els mateixos professors van assumir les despeses d'algunes pràctiques. Manca seguir estudiant l'impacte en l'educació en ciències d'aquesta modalitat dels TPL durant la pandèmia.

Paraules clau: treballs pràctics; pràctiques de laboratori; ciències naturals; pandèmia.

\section{Introducción}

Este es un artículo que se sitúa en la cotidianidad de las labores de enseñanza de los profesores, en cómo la pandemia "puso de cabeza" los rituales típicos de las prácticas de laboratorio en la escuela y en la universidad. 
Más allá de poner en evidencia la enorme desventaja educativa que hay entre los estudiantes que tienen acceso a internet, a equipos informáticos que funcionen, a espacios en su hogar adecuados para atender a su formación, frente a aquellos que no los tienen, este artículo señala, por un lado, los retos a los que se expusieron los profesores para ajustar su enseñanza a estas condiciones, intentando mantener los objetivos curriculares y por otro, la manera cómo los han venido enfrentando.

En el contexto particular de la enseñanza de las ciencias naturales, caracterizada por la utilización de laboratorios, equipos y reactivos, y por una concepción estandarizada de cómo desarrollarlos, el desafío es aún más grande. Y de la manera como los maestros respondan para su continuidad, aun en circunstancias de distanciamiento y de la no posibilidad de ir al edificio escolar, depende la valoración del impacto sobre el derecho a la educación que tienen niños, niñas y adolescentes.

De esta manera, el ajuste necesario se complejiza (o no), dependiendo de la concepción que los profesores tengan sobre el papel de las prácticas de laboratorio en la formación de sus estudiantes.

\section{La importancia del Trabajo Práctico de Laboratorio (TPL) en la educación en ciencias}

Las prácticas experimentales son consideradas como un recurso indispensable e integral para la enseñanza de las ciencias naturales (Canavarro-Benite y Machado-Benite, 2009), porque contribuyen a que el estudiante comprenda cómo opera el pensamiento científico y qué tipo de problemas resuelve, cómo es el lenguaje que usa e incluso, cómo avanza la ciencia. Desde este punto de vista, el TPL complementa la fundamentación teórica, el desarrollo de habilidades y capacidades de pensamiento y promueve la adquisición y perfeccionamiento de habilidades y destrezas necesarias para la actividad experimental (López-Rua y Tamayo-Alzate, 2012).

Caamaño (2003) dice que con los trabajos experimentales se logra alcanzar objetivos como la familiarización, observación e interpretación de hechos o fenómenos variados; además, pueden promover la contrastación de hipótesis; brindar destrezas en el manejo de equipos; le permite a los estudiantes aplicar diferentes estrategias y métodos de investigación en busca de la solución de problemas; y, por último, las prácticas de laboratorio promueven en los estudiantes la comprensión experimental de la ciencia (Sánchez Moreno et al, 2016).

Lacolla (2012) se refiere a que una de las representaciones sociales más asociada a la ciencia en sí misma es la de alguien trabajando en un laboratorio (sofisticado) en el cual tiene lugar la experimentación, relacionando a los científicos con imágenes estereotipadas de personas que "...trabajan solos y dentro de un laboratorio, usan delantal y gafas y producen reacciones llamativas y/o peligrosas." (p. 173).

Cuando se trae la pregunta por esa representación al campo educativo, los profesores y los estudiantes suelen aludir a un espacio particular dentro del edificio de la institución educativa, con grandes mesas, denominado 'laboratorio' en el que se guardan -cosas que muchas veces no se usan- en gavetas, materiales de vidrio, algunos instrumentos (balanzas, microscopios) y algunos preparados biológicos (un herbario, animales preservados en líquidos). A ese lugar van 
los estudiantes mayores (a los de preescolar y primaria casi nunca se les lleva porque corren peligro de herirse o porque rompen lo que hay allí y que es tan costoso).

Además, para realizar las prácticas de laboratorio se presentan muchos obstáculos -falta de espacio, poco material, tiempo reducido, un alto número de estudiantes, concepciones tanto de docentes como de estudiantes, etc.- (Siso-Pavón et al, 2010; Werner-Da Rosa y Becker-Da Rosa, 2010; Canavarro-Benite y Machado-Benite, 2009; Tamir y García-Rovira, 1992). Esta representación hace que la experimentación se vea muy limitada, y si a esta se le suman las condiciones de la pandemia, el logro de importantes metas para la educación científica se ve aún más difícil de alcanzar.

La situación de confinamiento ha complicado la posibilidad de que se lleven a cabo los trabajos experimentales en las escuelas, lo que trae como consecuencia una ruptura aún mayor entre la enseñanza de las ciencias y el papel del TPL en el pensamiento de los profesores y, que su importancia se debilite en la mente de los estudiantes y de los futuros profesores de ciencias.

Entonces, ¿qué hicieron los profesores de ciencias en Argentina y en Colombia? ¿Renunciaron a llevar a cabo las prácticas de laboratorio? ¿Se adaptaron a las circunstancias buscando soluciones? En este artículo se quiere describir la capacidad de los profesores de ciencias en ambos países, para sobreponerse a la situación. Esta característica de su saber profesional resulta extremadamente importante porque predice, que los profesores pueden adaptar su enseñanza a los cambios para privilegiar la consecución de las metas de la educación en ciencias.

Tal como señalan Morales et al. (2020, p. 6 ), los docentes "estamos acostumbrados a enfrentar situaciones problemáticas y encontrar soluciones creativas en beneficio de nuestros estudiantes" siendo el momento actual un contexto en el que se puede y debe asumir el desafío de "replantearnos nuestras prácticas como docentes... convertir debilidades en fortalezas y encontrar formas más adecuadas... de mejorar los procesos educativos en general". Así, se debe realizar una actividad reflexiva como un proceso consciente, intencional y metódico que signifique una atención explícita y detenida para pensar en lo que se hace y en las mejores maneras de intervenir (Cerecero Medina, 2018).

\section{Métodos y materiales}

Se trabajó con un grupo de profesores colombianos y otro grupo de profesores argentinos a partir de un muestreo por conveniencia (Hernández-Sampieri et al., 2014), donde profesores y egresados de ambos contextos participaron voluntariamente. En la Tabla 1 se presentan los datos que se recolectaron con el único propósito de caracterizar las muestras de profesores.

Entre abril y junio de 2020, los docentes que participaron del estudio respondieron un instrumento tipo formulario Google, sobre trabajo práctico de laboratorio (TPL) que involucró las siguientes preguntas: 1) ¿Cuál es su opinión en relación con los materiales (instrumentos, equipamiento y reactivos) y la realización de las prácticas experimentales?; 2) ¿Cómo ha planteado con sus estudiantes las prácticas experimentales durante la situación actual en la pandemia? y, 3) La situación vivida durante la pandemia en relación con la educación, ċle ha permitido replantearse la forma de trabajo experimental? ¿De qué manera? 


\begin{tabular}{|c|c|c|c|}
\hline & & Colombia & Argentina \\
\hline \multicolumn{2}{|l|}{ Número de profesores } & 32 & 50 \\
\hline \multirow[b]{2}{*}{ Título de pregrado } & Sin formación docente & 8 & 45 \\
\hline & Con formación docente & 27 & 8 \\
\hline \multirow{3}{*}{ Posgrado } & $\begin{array}{l}\text { Disciplinas científicas y } \\
\text { otras }\end{array}$ & 8 & 42 \\
\hline & Afines a la educación & 15 & 6 \\
\hline & Sin posgrados & 12 & 3 \\
\hline \multirow{3}{*}{ Nivel educativo en el que enseña } & Básica & 20 & \multirow{2}{*}{26} \\
\hline & Media & 8 & \\
\hline & Superior & 10 & 30 \\
\hline
\end{tabular}

Tabla 1. Descripción de profesores participantes.

Para poder entender fácilmente lo que se presenta en la Tabla 1, atendiendo a las diferencias entre los sistemas educativos de Colombia y Argentina, es importante describir aspectos generales de ambos sistemas. El sistema educativo colombiano está conformado por: la educación inicial, la educación preescolar, la educación básica (cinco grados en primaria y cuatro en secundaria), la educación media (dos grados y culmina con el título de bachiller), y la educación superior (Ministerio de Educación Nacional, 2019). En el caso del sistema educativo argentino, se diferencia la educación inicial ( 3 a 5 años de edad); la educación primaria (incluye seis o siete grados de acuerdo a la jurisdicción provincial); la educación secundaria (incluye cinco o seis años de acuerdo a la jurisdicción provincial) y la educación superior (universitaria y no universitaria) (Ley de Educación Nacional No 26.206).

El perfil de los profesores participantes indica que todos ellos tienen algún grado de formación en las disciplinas científicas (biología, física, química), aunque no todos han tenido formación en educación. En efecto, hay quienes tienen titulaciones que combinan la fundamentación científica con la educativa, pero hay otros que optan por avanzar en esta última durante el posgrado.

La tabla también muestra que los profesores de estos países se desempeñan en un amplio espectro en lo que respecta al nivel educativo: los rasgos socioeconómicos de los profesores argentinos y colombianos describen personas que distribuyen su tiempo entre las labores en una escuela (desde el preescolar hasta el secundario) y en la universidad o en otros espacios educativos.

Lo que todos tienen en común es que enseñan ciencias naturales y han tenido que enfrentar dilemas cuando piensan en cómo hacer el TPL sin tener ese espacio físico. 


\section{Resultados}

Las respuestas de los profesores permiten "ver con su mirada" el panorama de la educación (en todos sus niveles, incluidos los de formación de futuros profesores) en el contexto actual y en otro de pospandemia. A continuación, se presenta lo que respondieron a cada una de las preguntas.

\section{1 ¿Cuál es su opinión en relación con los materiales (instrumentos, equipamiento y reactivos) y la realización de las prácticas experimentales?}

Como puede observarse en la Figura 1, en lo que respecta a la opinión de los profesores en relación con los materiales, una quinta parte de los colombianos consideran que se requieren materiales específicos para poder realizar las prácticas de laboratorio y así tener resultados ya definidos, pero es mayor el número de estos profesores que consideran que es posible realizar estas actividades haciendo uso de materiales que se encuentren a la mano de los propios docentes o estudiantes. En cuanto a los profesores argentinos, se cumple la misma tendencia que presentan los colombianos con relación a los instrumentos o equipamiento utilizado en las prácticas experimentales, pero un menor porcentaje de profesores argentinos consideran que son necesarios materiales concretos para llevar a cabo estas actividades.

Por otro lado, pasando a abordar la realización de las prácticas experimentales, es menor el porcentaje de profesores colombianos, en relación con los argentinos, que consideran que, si no se posee un material o equipamiento adecuado, no se pueden realizar las prácticas de laboratorio. Continuando, pocos profesores argentinos piensan que la realización o no de las prácticas depende de los objetivos que se presenten en la asignatura del pregrado en específico (esto para instituciones de educación superior) o en las instituciones de educación secundaria, pero los profesores colombianos no presentaron reflexiones con respecto a esto. Para finalizar con esta pregunta, un mínimo porcentaje de docentes de Colombia menciona que para poder realizar las prácticas experimentales se necesita hacer fusión de varias alternativas, pero, en cambio, los profesores de Argentina no se plantean cuestiones relacionadas a esto.

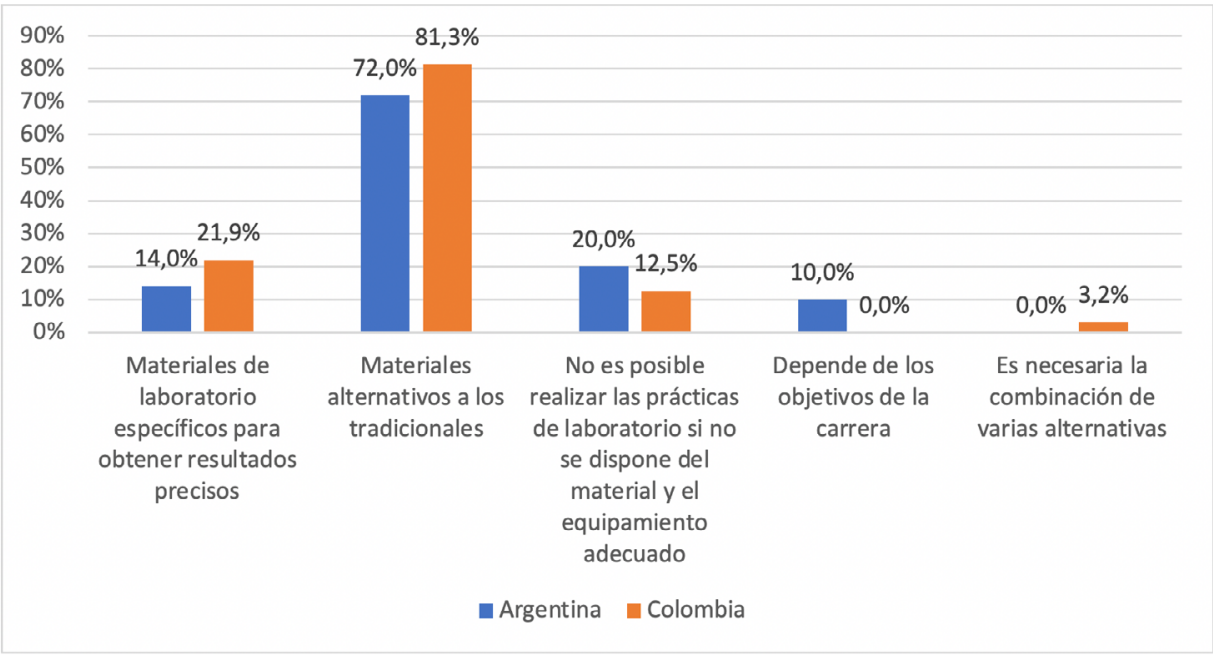

Figura 1. ¿Cuál es su opinión en relación con los materiales (instrumentos, equipamiento y reactivos) y la realización de las prácticas experimentales? 


\section{2 ¿Cómo ha planteado con sus estudiantes las prácticas experimentales durante la situación actual en la pandemia?}

Como se ilustra en la Figura 2, los profesores lograron adaptarse a las condiciones del trabajo en casa, diseñando prácticas de laboratorio a partir de insumos que se encuentran en los hogares, dando prioridad a las finalidades de la educación en ciencias como la observación, el análisis de los resultados, la sistematización de la información y el desarrollo de la capacidad reflexiva y crítica que entraña la formación científica.

Al respecto dos profesores argentinos puntualizan lo siguiente:

En la pandemia traté de focalizarme en experiencias de indagación sencillas, con materiales que resulten fáciles de conseguir y lo más económico posible. Estas prácticas, además, intenté orientarlas para que indaguen en torno a la ciencia de lo cotidiano, que tomen sus casas como laboratorios. Es importante destacar que doy Ciencias Naturales a niños de 11 y 12 años.

Si, creo que [la pandemia] ha permitido un descubrimiento conjunto de alumno y profesor de nuevas técnicas de enseñanza para impartir los conocimientos, sobre todo las actividades experimentales que han debido ser parcialmente reemplazadas por material audiovisual.

No obstante, algunos docentes mencionan que más allá de la capacidad de adaptación, existen barreras insalvables que tienen que ver con los recursos con los que cuentan algunos hogares, relacionados con el acceso a internet y dispositivos para el uso de los niños. Al respecto un profesor colombiano menciona:

El trabajo experimental durante la pandemia ha posibilitado otorgarle más autonomía a los estudiantes y un mejor aprovechamiento de los materiales existentes en el entorno. Sin embargo, debido a que la población estudiantil con la que trabajo presenta bajas condiciones económicas, es decir, muchos de ellos no cuentan con internet o aparatos tecnológicos, no ha sido posible proponer trabajos experimentales por medio de simulaciones, laboratorios virtuales, entre otros.

A pesar de que la pandemia afecta la salud de todos los seres humanos, el impacto sobre las distintas actividades de la sociedad es diferente para cada individuo dada su condición socioeconómica. Los niños, niñas y adolescentes provenientes de medios desfavorecidos no cuentan con servicios básicos como acceso a internet $y / o$ la disponibilidad de un equipo de cómputo, lo que limita ejercer su derecho a la educación:

En ámbitos rurales los docentes nos hemos tenido que reinventar aún más, no tenemos medios tecnológicos y en muchos casos la comunicación es imposible. El uso de medios tradicionales y del papel ha sido herramienta primordial.

Algunos docentes reportan que la única forma de comunicación que tuvieron con un importante número de estudiantes fue a través de guías impresas que se hacían llegar a través de amigos o familiares y que su implementación dependía del acompañamiento de los padres o cuidadores. 


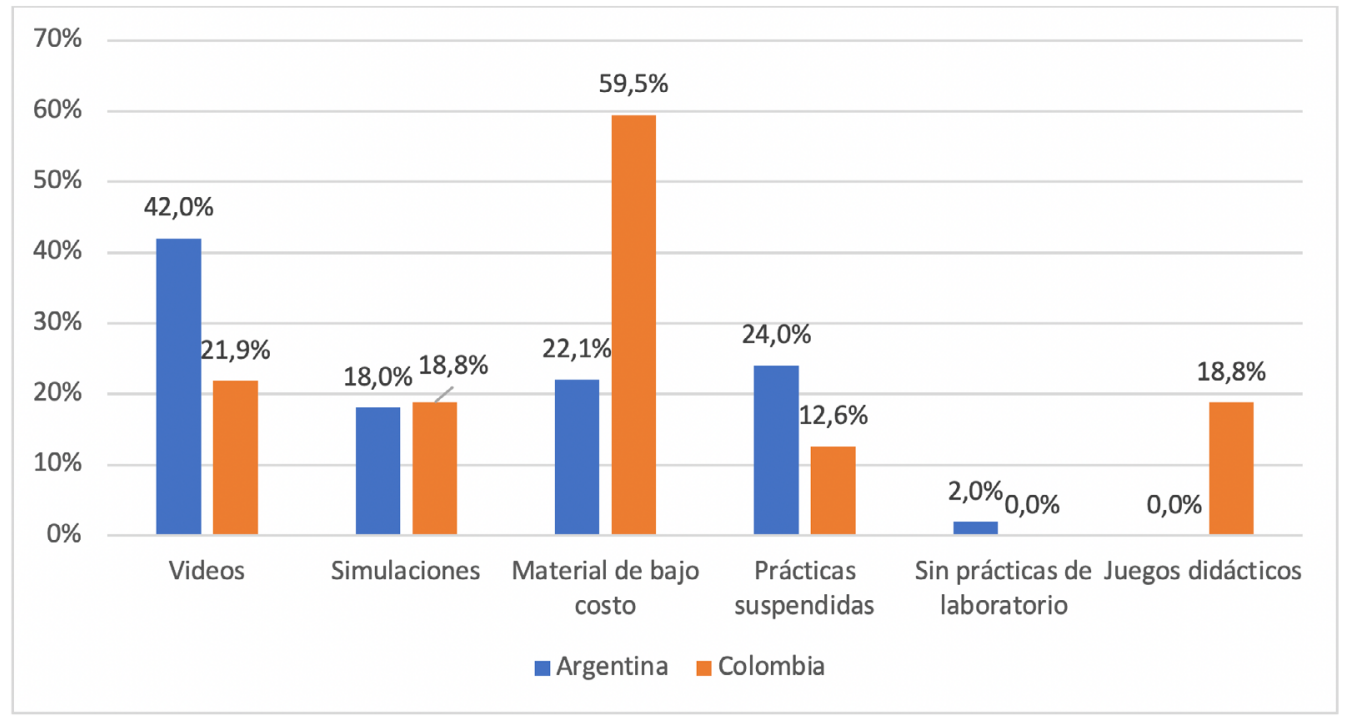

Figura 2. ¿Cómo ha planteado con sus estudiantes las prácticas experimentales durante la situación actual en la pandemia?

Se puede apreciar en la Figura 2 que son pocos los profesores que tienen a cargo cursos sin prácticas experimentales. Con relación a los profesores que en la normalidad si realizan prácticas experimentales, casi una cuarta parte de los profesores argentinos suspendieron estas actividades en tiempos de pandemia, y aunque en Colombia es menor ese porcentaje, también es un valor bastante alto. La suspensión de actividades se evidenció especialmente en el nivel superior, al respecto un profesor señaló:

En la facultad trabajamos muchísimo con material vegetal y microscopios, y si bien podemos proporcionarles a los alumnos fotos, atlas interactivos, etc., no podrán adquirir este año las destrezas necesarias para colocar un preparado histológico, enfocarlo correctamente, utilizar varios aumentos, etc.

Por otra parte, se presentan diferentes propuestas o alternativas para llevar a cabo las prácticas experimentales durante la pandemia. En cuanto a los profesores argentinos, las principales estrategias que propusieron fueron los videos, como la más utilizada, y el uso de materiales de bajo costo o que se encuentren en el lugar donde habitan cada uno de los estudiantes; y, con un menor porcentaje, se presentan las simulaciones como estrategias aplicadas por los argentinos. Por el lado de los docentes colombianos, también se presentan los videos y los materiales de bajo costo como los más utilizados, pero son estos últimos los que predominan con más de la mitad de los docentes aplicando esta estrategia. Finalmente, al igual que los compañeros argentinos, son las simulaciones las que presentan un menor porcentaje, pero aun siendo significativas en ambos contextos.

\subsection{La situación vivida durante la pandemia en relación con la educación, ¿̇e ha permitido replantearse la forma de trabajo experimental? ¿De qué manera?}

La Figura 3 muestra que la mayoría de los profesores Sí se han replanteado la forma de hacer TPL, tanto en Argentina como en Colombia. Por otra parte, un pequeño porcentaje de profesores colombianos se replantearon acerca de características de la enseñanza diferentes a los trabajos experimentales. 


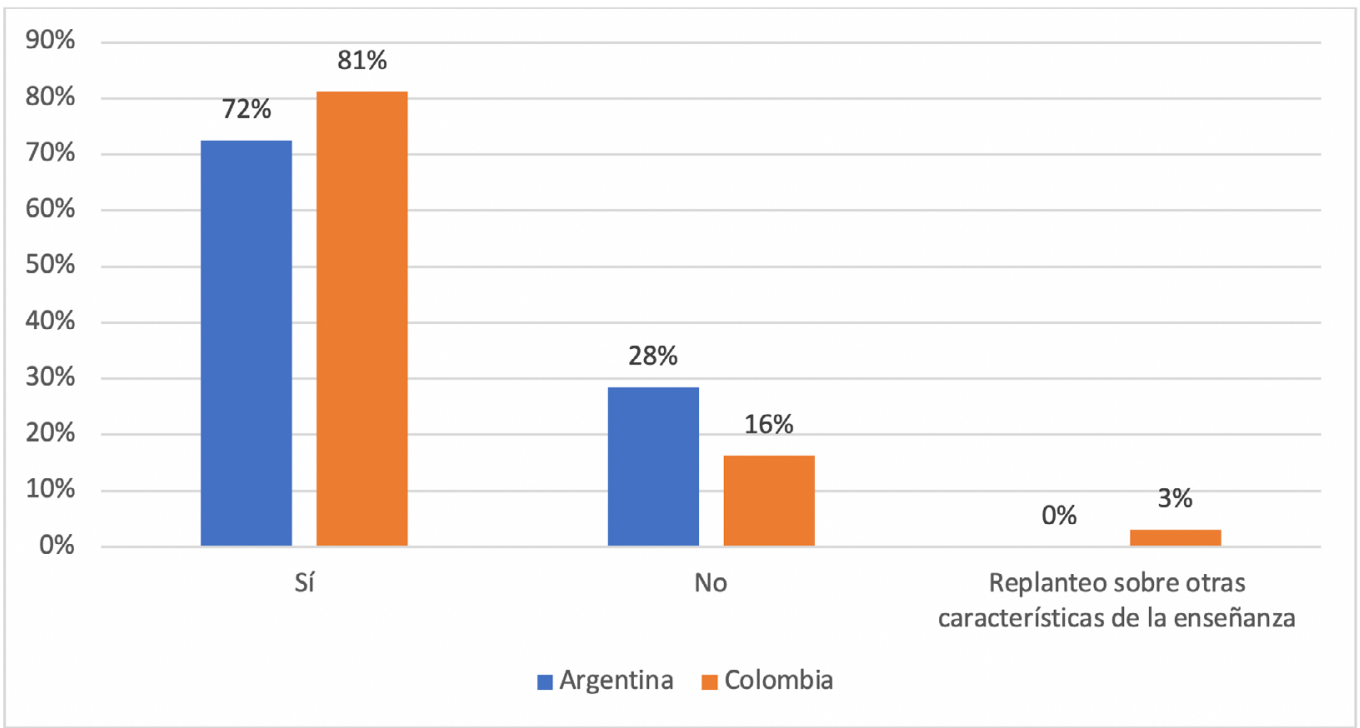

Figura 3. La situación vivida durante la pandemia en relación con la educación, ¿le ha permitido replantearse la forma de trabajo experimental?

En la Tabla 2 se muestran las razones de quienes afirmaron que NO se replantearon la forma de trabajo experimental:

\begin{tabular}{|c|c|}
\hline Argentina & Colombia \\
\hline $\begin{array}{l}\text { Para estudiar la anatomía de plantas necesitan si o si un } \\
\text { microscopio si es que queremos que el alumno fortalezca } \\
\text { las habilidades de preparación del material y observación } \\
\text { bajo microscopio. }\end{array}$ & Por no ser necesario. \\
\hline $\begin{array}{l}\text { [...] Muchas actividades quedaron a la espera de la vuelta } \\
\text { a la presencialidad. }\end{array}$ & $\begin{array}{l}\text { Por no ser posible considerar otra opción a la } \\
\text { presencial. }\end{array}$ \\
\hline $\begin{array}{l}\text { Creo que es muy difícil llevar a cabo trabajos } \\
\text { experimentales en la virtualidad, y no he encontrado una } \\
\text { forma de solucionar este problema. }\end{array}$ & Por la forma habitual de trabajo. \\
\hline Algunos [TPL] es necesario realizarlos en presencialidad. & $\begin{array}{l}\text { Desde mi práctica he tratado de hacer las experiencias } \\
\text { de laboratorio con implementos y situaciones de la vida } \\
\text { cotidiana, por lo cual no ha sido muy diferente ahora. }\end{array}$ \\
\hline $\begin{array}{l}\text { [...] Es medio complicado que los alumnos aprendan los } \\
\text { conceptos prácticos si ellos no realizan los TPL } \\
\text { correspondientes. }\end{array}$ & $\begin{array}{l}\text { En parte no, porque he sido partidaria de que cualquier } \\
\text { espacio puede convertirse en laboratorio. De hecho, } \\
\text { considero que esto permite que [los estudiantes] vean } \\
\text { las ciencias naturales en todo. }\end{array}$ \\
\hline
\end{tabular}

Tabla 2. La situación vivida durante la pandemia en relación con la educación, ¿le ha permitido replantearse la forma de trabajo experimental? 
Las respuestas de estos profesores no pueden interpretarse en modo alguno como una imposibilidad de adaptarse a las nuevas circunstancias, al considerar sus argumentos. Al respecto señalan que hay TPL que deben suspenderse para esperar a la presencialidad, por la necesidad de que los estudiantes comprendan que buena parte del conocimiento científico se genera en tanto es posible manipular muchas variables propias de los fenómenos naturales, para crear una situación artificial de tipo experimental, y esto se alcanza al llevar al estudiante al espacio físico del laboratorio, donde hay instrumentos que son parte del entramado esencial de relaciones que identifican a quienes se dedican a hacer ciencia.

La "práctica" del TPL coloca al estudiante en situación de vivenciar qué son y cómo funcionan los instrumentos, qué características tienen los materiales, cómo se llaman, cómo y de qué se habla en el laboratorio, cómo hay que comportarse allí dentro o por qué hay que vestirse de otra manera cuando se está en ese lugar. La comunidad de quienes "practican" las ciencias estudiando fenómenos naturales, algunos de los cuales son susceptibles de ser sometidos a la experimentación, se vale del espacio educativo denominado "laboratorio" para comunicar a los aprendices una parte muy importante de lo que es su identidad y de ciertos rasgos que caracterizan la cultura científica.

Para lograrlo, desde una mirada curricular, el TPL promueve el aprendizaje de procedimientos con el concomitante desarrollo de habilidades y destrezas, además de que complementa los contenidos conceptuales que tienen lugar en la clase. Ambos TPL y clase son necesarios para ayudar al estudiante a desarrollar sus capacidades de pensamiento, aquellas que son tan particulares de la ciencia, pero que todo ciudadano necesita entender en tanto es una meta en la democratización de la educación en ciencias.

Los profesores que respondieron que no replantearían su forma de trabajo experimental por efectos de la pandemia, se alejan mucho de ser 'malos' profesores. Al contrario, Ilaman la atención porque comprenden importantísimas implicaciones de la práctica experimental para la educación en ciencias naturales, especialmente en la educación superior.

Hay otras respuestas negativas que son relevantes porque aluden a una posibilidad real que no había sido planteada por los investigadores con anterioridad. La pregunta era: "La situación vivida durante la pandemia en relación con la educación, ¿le ha permitido replantearse la forma de trabajo experimental?" Los profesores respondieron "No" porque desde antes de la pandemia ya se habían dado a la tarea de hacer esos replanteamientos (p. ej., por la forma habitual de trabajo).

Este tipo de profesores recuerda a aquellos que han llegado a escuelas donde los recursos son tan limitados, que de lo único que pueden echar mano para educar a sus estudiantes, es de lo que hay a su alrededor. Estos profesores se comprometen estrechamente con su trabajo, pero también son muy originales y creativos, porque su comprensión sobre las implicaciones del TPL para la educación de sus estudiantes les ayuda a buscar la manera de reemplazar las prácticas habituales basadas en guías que implican materiales e instrumentos sofisticados, por otros que, debidamente organizados, se dirigen a la misma meta: servir a sus estudiantes de andamiaje (Wood et al., 1976) para entender por qué la ciencia es un tipo especial de conocimiento en el cual, ciertas afirmaciones pueden ser cuestionadas por la evidencia empírica mediante la actividad experimental. 
Los profesores que respondieron que la pandemia les había hecho replantear su forma de TPL, también citaron razones sumamente interesantes. Algunas de ellas fueron las que se citan en la Figura 4:

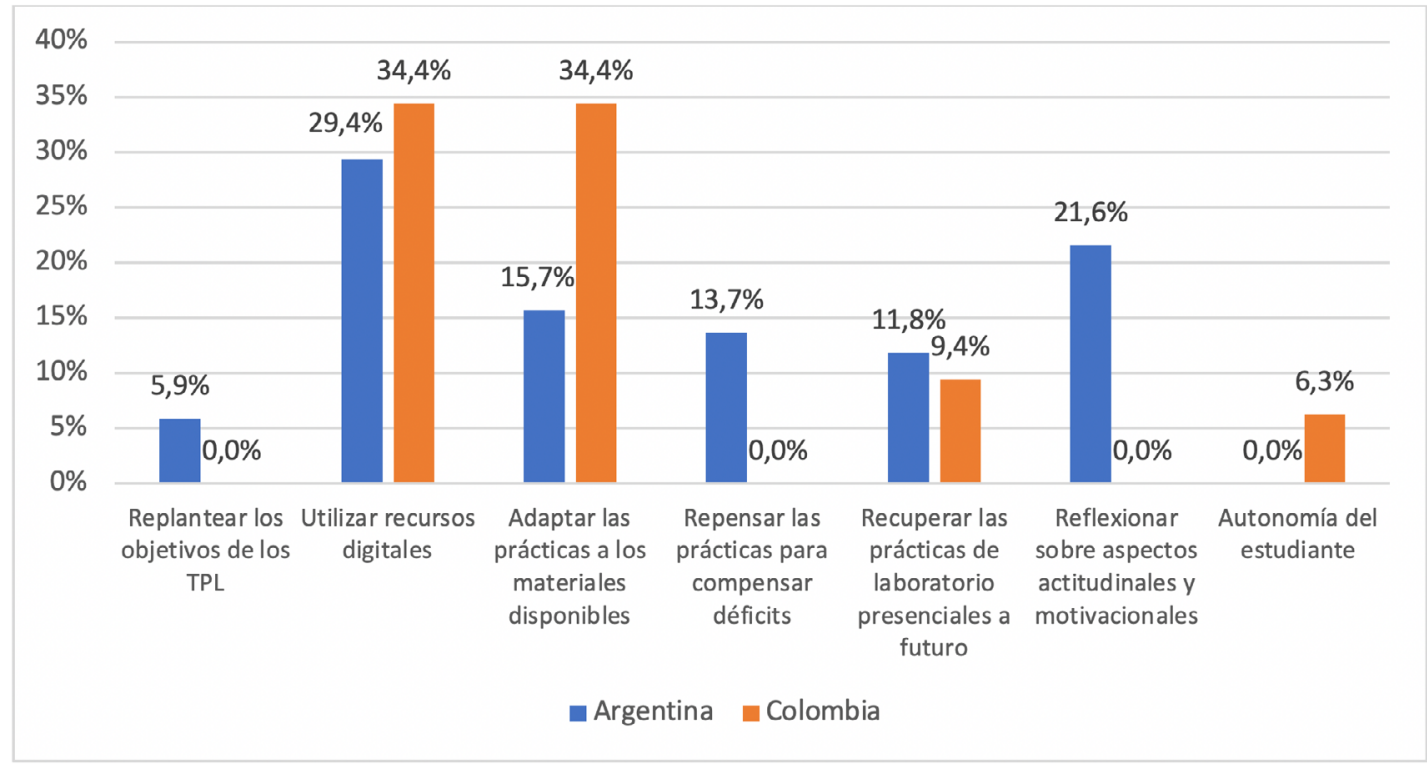

Figura 4. ¿De qué manera se ha replanteado la forma de trabajo experimental?

En la Figura 4, se observan las respuestas de los profesores ya agrupadas en categorías que permiten compararlas. Para la muestra colombiana, los profesores mencionan, en primer lugar, que principalmente se han adaptado utilizando con sus estudiantes recursos digitales, en el segundo lugar se encuentra el uso de herramientas o implementos que se encuentren a la mano de ellos mismos o de sus estudiantes. Por otro lado, un considerable porcentaje de profesores expresa que las prácticas de laboratorio se recuperen en un futuro, posiblemente, cuando la situación de pandemia culmine, para finalizar con los docentes colombianos, aproximadamente el 5\% de estos dejaron el trabajo experimental a la autonomía del estudiante.

Pasando a los profesores argentinos, estos presentan una mayor cantidad de formas de replantearse el trabajo experimental; al igual que los docentes colombianos, la más popular es realizar las prácticas haciendo uso de diferentes recursos digitales, luego los profesores afirman que han adaptado la propuesta teniendo en cuenta aspectos actitudinales y motivacionales de los estudiantes. Los profesores colombianos y argentinos coinciden en el uso de diferentes materiales disponibles para adaptar las prácticas y, como última estrategia, el repensar las prácticas para compensar diferentes déficits que se presentaron debido a la situación de anormalidad académica tanto en el contexto local como mundial. Con relación a los profesores argentinos que optaron por recuperar las prácticas de laboratorio presenciales a futuro, este porcentaje fue menor que el de los profesores colombianos. 
Como se mencionó antes, los profesores de ambos países coinciden en que ese replanteamiento implica utilizar recursos digitales y adaptar las prácticas a los materiales disponibles y lo hacen de la siguiente manera:

\begin{tabular}{|c|c|}
\hline Argentina & Colombia \\
\hline \multicolumn{2}{|c|}{ Utilizar recursos digitales } \\
\hline $\begin{array}{l}\text { Es evidente que las TIC son un buen soporte para suplir estas } \\
\text { carencias. }\end{array}$ & $\begin{array}{l}\text { Una [de las difícultades de no tener laboratorio] es } \\
\text { la manipulación de objetos. Solo [se soluciona] } \\
\text { con la observación de videos o applet en los que se } \\
\text { dan en algunas ocasiones situaciones similares a } \\
\text { las reales. }\end{array}$ \\
\hline $\begin{array}{l}\text { Creo que [la pandemia] ha permitido un descubrimiento } \\
\text { conjunto de alumno y profesor de nuevas técnicas de } \\
\text { enseñanza para impartir los conocimientos, sobre todo las } \\
\text { actividades experimentales que han debido ser parcialmente } \\
\text { reemplazadas por material audiovisual. }\end{array}$ & $\begin{array}{l}\text { Reinventándome como docente y buscando } \\
\text { estrategias } y / o \text { herramientas virtuales. }\end{array}$ \\
\hline $\begin{array}{l}\text { Buscar videos, material multimedia en redes y clases en zoom } \\
\text { o meet; aulas virtuales. }\end{array}$ & $\begin{array}{l}\text { De forma más didáctica integrando las tecnologias } \\
\text { y sus alcances. }\end{array}$ \\
\hline $\begin{array}{l}\text { He podido dar los contenidos valiéndome de recursos } \\
\text { tecnológicos como fotografias, etc. }\end{array}$ & $\begin{array}{l}\text { Haciendo uso de las potencialidades de las } \\
\text { herramientas digitales. }\end{array}$ \\
\hline $\begin{array}{l}\text { Hay prácticas experimentales que pudieron ser mostradas con } \\
\text { videos o simulaciones; y actividades de mini trabajos prácticos } \\
\text { grabados en vivo, y también de simulación. }\end{array}$ & $\begin{array}{l}\text { En la educación secundaria he promovido los } \\
\text { laboratorios tipo simulación. }\end{array}$ \\
\hline \multicolumn{2}{|c|}{ Adaptar las prácticas a los materiales disponibles } \\
\hline Me adapté a lo que tengo en mi entorno. & $\begin{array}{l}\text { En la búsqueda de experimentos más prácticos que } \\
\text { sean posibles de realizar desde la comida de la } \\
\text { casa. }\end{array}$ \\
\hline $\begin{array}{l}\text { Buscar realizar la práctica con materiales que tengan en la casa } \\
\text { y que sean de bajo costo. }\end{array}$ & $\begin{array}{l}\text { Porque dependemos de los espacios físicos y lo } \\
\text { que se busca es que el estudiante se dé cuenta } \\
\text { de que en su hogar puede convertirse en un } \\
\text { científico que explora múltiples posibilidades. }\end{array}$ \\
\hline $\begin{array}{l}\text { En las ciencias ambientales se pueden realizar experimentos } \\
\text { con elementos simples que tenemos en los hogares, por eso } \\
\text { pude sobrellevar este problema. }\end{array}$ & $\begin{array}{l}\text { Se pueden hacer prácticas experimentales en casa } \\
\text { con materiales diferentes o alternativos. }\end{array}$ \\
\hline $\begin{array}{l}\text { En la pandemia traté de focalizarme en experiencias de } \\
\text { indagación sencillas, con materiales que resulten fáciles de } \\
\text { conseguir y lo más económicos posible. Estas prácticas, } \\
\text { además, intenté orientarlas para que indaguen en torno a la } \\
\text { ciencia de lo cotidiano, que tomen sus casas como laboratorios. } \\
\text { Es importante destacar que doy Ciencias Naturales a niños de } \\
11 \text { y } 12 \text { años. }\end{array}$ & $\begin{array}{l}\text { Tratando de generar espacios caseros, pero que la } \\
\text { práctica se realice con supervisión de los padres y } \\
\text { el apoyo sincrónico o asincrónico. }\end{array}$ \\
\hline $\begin{array}{l}\text { Teniendo que adaptar las reacciones a materiales de uso diario } \\
\text { y bajo costo. }\end{array}$ & $\begin{array}{l}\text { Mis estudiantes en su totalidad carecen de lo } \\
\text { básico, incluso para permitirse un acceso constante }\end{array}$ \\
\hline
\end{tabular}

Tabla 3. Razones de usar recursos digitales y materiales disponibles. 
Las respuestas de los profesores indican que la pandemia fue una oportunidad para reinventar su docencia, buscando en internet recursos que quizás no hubiesen usado en situación de normalidad. Sin embargo, son conscientes de las limitaciones de estos recursos:

En ámbitos rurales los docentes nos hemos tenido que reinventar aún más, no tenemos medios tecnológicos y en muchos casos la comunicación es imposible. El uso de medios tradicionales y del papel han sido herramientas primordiales.

La situación actual nos deja ver la realidad de la mayoría de personas; mis estudiantes en su totalidad carecen de lo básico, incluso para permitirse un acceso constante y significativo a la educación, las formas de trabajo experimental suelen hacerse con materiales de bajo costo, en su mayoría costeados por el docente para cada estudiante.

Hay que reevaluar los objetivos de cada materia, ya que el tiempo que les toma a los alumnos aprender [con recursos digitales], es diferente a la que les toma de manera presencial. El material que les brindemos tiene que obedecer a lo que uno espera que aprendan, pero no excederse, ya que de esa forma se sienten más agobiados. En cuanto a las prácticas de laboratorio lamentablemente no pueden ser reemplazadas en esta virtualidad si deseamos que la calidad del egresado, en cuanto a desempeño práctico, no decaiga.

Estos comentarios incluyen explicaciones de los profesores sobre las razones por las cuales cuestionan el potencial de los recursos digitales. Sin duda alguna, ellos tienen claro que el TPL tiene unos objetivos curriculares cuyo alcance se ve limitado por las posibilidades de la virtualidad. Hay prácticas que son muy importantes en términos del aporte a la formación disciplinar del estudiante, que definitivamente tienen que ser aplazadas hasta que esté disponible el espacio físico del laboratorio para crear las condiciones en las que el estudiante desarrolle ciertas habilidades y destrezas experimentales.

Las explicaciones incluyen argumentos que señalan las condiciones socioeconómicas que obstaculizan una educación de mejor calidad en nuestros países. Sólo hay que ver la respuesta del profesor que se refiere a los contextos rurales (algunos ni siquiera tienen luz eléctrica. iMenos aún señal de internet!), con lo cual, es evidente la desventaja de sus estudiantes frente a otros que sí pueden acceder a estos medios comunicativos, así sea esporádicamente. Tal situación se suma a la disponibilidad de recursos para la subsistencia: Los profesores cubren en muchas oportunidades los costos de los materiales para sus clases y TPL. De ahí que se está evidenciando un bello acto de generosidad de parte de educadores sumamente comprometidos con ofrecer a sus estudiantes lo que está a su alcance para seguir adelante.

Los profesores de Argentina fueron más precisos en señalar algunas características de las formas como han replanteado sus prácticas. No quiere decir que los colombianos no hayan aludido a ellas en sus respuestas, sino que fueron menos explícitos. Estas otras características son muy importantes a efectos de describir la capacidad de los profesores de ciencias en ambos países, para sobreponerse a la situación de confinamiento o de limitado acceso al laboratorio. A continuación, se presentan algunos ejemplos de cómo lo hicieron:

\subsubsection{Replantear los objetivos del TPL}

Las investigaciones sobre formación de profesores de ciencias naturales muestran que los docentes que llegan a comprender las implicaciones de la educación de sus estudiantes hacen ajustes a su planificación: cambian el nivel de complejidad, dan directrices para que el 
estudiante trabaje de manera individual, son sensibles a las diferencias entre la experiencia de aprendizaje acompañado por el profesor y la que es individual y se cuestionan sobre el papel de los contenidos. Estos profesores se comportan metacognitivamente (Angulo, 2002). Al respecto, algunos de los profesores participantes en este estudio señalan:

\begin{abstract}
Hay que planificar las actividades para reducir la complejidad y que sean autoguiadas para fomentar la autonomía de los estudiantes.
\end{abstract}

Hay que reevaluar los objetivos de cada materia, ya que el tiempo que les toman a los alumnos para aprender es diferente a la que les toma de manera presencial.

La necesidad de buscar nuevas herramientas pedagógicas para poder lograr el proceso de enseñanza aprendizaje me ha permitido replantearme mis prácticas e incluso el contenido de la asignatura.

\title{
3.3.2 Repensar las prácticas para compensar déficits
}

Esta categoría de respuestas reúne ciertas explicaciones que complementan las razones por las cuales los profesores consideran que el TPL es irremplazable por otros recursos. Se puede ver:

Si bien soy docente de materia teórica, mis alumnos de Microbiología hacen el taller de dicha materia en otro turno. El dictado de los mismos [talleres] se vio muy afectado. Es imprescindible que los alumnos manejen instrumentos, técnicas y equipos y los vídeos no son suficientes, por lo que considero que al retornar lo más adecuado sería dictar algunas prácticas orientativas en el taller sin nota cuantitativa.

He ayudado a plantear el uso de aulas virtuales en complemento a las actividades presenciales para ciertas actividades.

[Para compensar la carencia del laboratorio propongo] Interpretación de observaciones, hipótesis, resultados y conclusiones a partir del análisis de textos de investigaciones realizadas. $O$ a partir de experimentos históricos.

Repensar las prácticas para compensar el hecho real de no poder contar con el espacio físico del laboratorio, pasa por admitir que en algunos casos -que implican desarrollar ciertos contenidos a fin de lograr determinados objetivos curriculares-, no hay otra opción sino la de esperar a que el control de la pandemia permita regresar a las instituciones educativas. La alternativa mientras eso ocurre, es compensar el TPL con lo que hay en la literatura, como, por ejemplo, analizar textos de investigaciones realizadas o documentos históricos que relatan experimentos. A este último respecto, la historia de la ciencia parece una buena fuente de recursos.

\subsubsection{Recuperar las prácticas a futuro}

Esta categoría de respuestas también se relaciona mucho con las razones a favor de ir en algún momento al laboratorio a desarrollar los TPL. Estos profesores lo ilustran en sus comentarios:

[...] buscando formas alternativas de actividades prácticas donde el estudiante pueda aprender y comprender procesos biológicos. También es cierto que muchas actividades quedaron a la espera de la vuelta a la presencialidad.

Si [debo replantear los TPL] pero no sé de qué manera porque para estudiar la anatomía de plantas [los estudiantes] necesitan si o si un microscopio, si es que 
queremos que el alumno fortalezca las habilidades de preparación del material y observación bajo microscopio.

Estos profesores sintetizan en sus comentarios las aproximaciones conceptuales sobre la importancia del TPL, a las que hicimos referencia al comenzar este artículo y que se han reiterado en otros momentos. Así los comentarios evidencian que hay unos propósitos de formación en la cultura científica que corresponden, por un lado, a ciertos modos en los que este conocimiento se construye y, por otro, a las estrategias mediante las cuales, la comunidad de practicantes de la ciencia forma a sus aprendices. Nos referimos a los procedimientos de la experimentación que son propios de cada ciencia.

\subsubsection{Reflexionar sobre aspectos actitudinales y motivacionales}

Al igual que pasa en las clases y en el laboratorio en tiempos sin pandemia, las actitudes y las motivaciones que los profesores logren promover en sus estudiantes resultan imprescindibles para comprometerlos con sus propios aprendizajes, en busca de la meta suprema del aprendizaje autónomo. Los replanteos que los profesores se hicieron tocaron aspectos como los siguientes:

Según el contexto, más el desarrollo de habilidades creativas, pues si no hay creatividad [los estudiantes] se ajustan a solo lo que hay en la guía sin salir del molde.

Buscar maneras creativas para despertar el interés y la indagación en los contenidos.

Buscar las formas de que los alumnos logren adquirir la experiencia de realizar un experimento, sin poder estar al lado de ellos para guiarlos.

Considero que lo más destacado en relación a eso es... que debemos ser un poco más recursivos y efectivos a la hora de entregar el mensaje que se pretende transmitir.

\section{Conclusiones}

Los profesores participantes mostraron cuál es su visión sobre el TPL en tiempos de pandemia. Para unos pocos, no hay opción distinta a la de esperar a volver al laboratorio porque hay prácticas que se deben llevar a cabo en ese espacio para aproximar a los estudiantes al logro de objetivos curriculares específicos, particularmente en educación superior.

Para la mayoría de ellos e indistintamente de sus países de origen, la pandemia suscitó un replanteamiento de los contenidos y estrategias vinculados al TPL, especialmente en quienes se desempeñan en básica y media. Acudieron a los recursos digitales (videos, aplicaciones, simulaciones) y también, a los materiales de fácil acceso y bajo costo con los que pueden contar sus estudiantes. Este tiempo, colocó a los profesores en situación de adaptar sus estrategias de enseñanza de manera muy rápida y para algunos de ellos hubo aprendizajes interesantes.

Los profesores son sensibles (y mucho) a las implicaciones de las condiciones socioeconómicas de sus estudiantes, porque si estas son escasas, limitan su acceso a equipos informáticos e internet. Pero también lo son ante las dificultades que tienen para conseguir algún recurso que reemplace el TPL. Entonces es allí cuando son los mismos profesores quienes asumen los costos, privilegiando la posibilidad de que sus estudiantes puedan educarse, pero 
hacen interesantes reflexiones sobre lo que tendría que hacerse a futuro, poniendo énfasis en las necesidades de replantear la formación inicial docente.

Lo que hay que preguntarse ahora, es cuál es el efecto que a futuro tendrá esta visión de los profesores sobre el TPL, cuando la pandemia permita reanudar las formas habituales de trabajo. Es fácil inferir de nuestros resultados que, en Argentina y Colombia, profesores como quienes participaron en el estudio que se presenta en este artículo, adopten estrategias que combinen de manera más explícita los recursos digitales a sus TPL. También estarán (aunque seguramente en menor medida) quienes decidan volver totalmente a las rutinas presenciales del laboratorio. Sea cual sea la decisión, es necesario e importante poder capitalizar lo aprendido, analizando lo que pasó en estos tiempos con la educación en ciencias de las nuevas generaciones y las consecuencias que trae para la promoción de la ciencia, la tecnología y la innovación en este lado del mundo.

\section{Referencias}

Angulo, F. (2002). Aprender a enseñar ciencias. Análisis de una propuesta para la formación inicial del profesorado de secundaria, basada en la metacognición. [Tesis doctoral. Universidad Autónoma de Barcelona, España]. http://hdl.handle.net/10803/4693

Caamaño, A. (2003). Los trabajos prácticos en ciencias. Editorial Graó.

Canavarro-Benite, A.M. y Machado-Benite, R.C. (2009). O laboratório didático no ensino de química: uma experiencia do ensino público brasileiro. Revista Iberoamericana de Educación, 48(2), 1-10. https://bit.ly/3GdZPJp

Cerecero Medina, I. (2018). Propuesta de un nuevo modelo: Práctica Reflexiva Mediada. Innoeduca. International Journal of Technology and Educational Innovation, 4(1), 44-53. https://doi.org/hd4b

Hernández-Sampieri, R.; Fernández-Collado, C. y Baptista-Lucio, P. (2014). Metodología de la investigación (6.a ed.). Editorial Mc Graw Hill.

Lacolla, L. (2012). La representación social que los estudiantes poseen acerca de las reacciones químicas y su incidencia en la construcción del concepto de cambio químico [Tesis doctoral. Universidad de Burgos, España].

Ley de Educación Nacional No 26.206. (2006). República Argentina. Consultado el 10 de junio de 2021. https://bit.ly/3KXdaJI

López-Rúa, A. M., Tamayo-Alzate, O. E. (2012). Las prácticas de laboratorio en la enseñanza de las ciencias naturales. Revista Latinoamericana de Estudios Educativos, 8(1), 145-166.

Ministerio de Educación Nacional. (2019, 03 de abril). Sistema Educativo Colombiano. Consultado el 10 de junio de 2021. https://bit.ly/3reo1Ek

Morales, L. M., Zorrilla, E. G. y Mazzitelli, C.A. (2020). Repensando nuestra práctica docente: la evaluación, una instancia del proceso educativo de la que no podemos prescindir. Boletín Educar en la incertidumbre: $7 .^{\mathrm{a}}$ edición. Universidad de Antioquia.

Sánchez-Moreno, A., Jaimes-Gómez, O., Jiménez-Rojas, F., Magallán-Muñoz, C.O. y Álvarez-López, J.L. (2016). Diseños experimentales caseros para la enseñanza de conceptos electromagnéticos en el Tecnológico Nacional de México. Revista Iberoamericana de Educación, 7o(2), 45-62. https://doi.org/hfds

Siso-Pavón, Z., Briceño, S., Alvarez, C. y Arana, J. (2010). Las prácticas de laboratorio en la formación del profesorado en química. Un primer acercamiento. Revista Electrónica Diálogos Educativos, 9(18).

https://bit.ly/309JEqj 
Tamir, P., García-Rovira, M. P. (1992). Características de los ejercicios de prácticas de laboratorio incluidos en los libros de texto de ciencias utilizados en Cataluña. Enseñanza de las Ciencias, 1O(1), 3-12. https://bit.ly/3IUlRD4

Werner-Da Rosa, C. y Becker-Da Rosa, A. (2010). Discutindo as concepções epistemológicas a partir da metodologia utilizada no laboratório didático de Física. Revista Iberoamericana de Educación, 52(6), 1-11. https://doi.org/hfdt

Wood, D., Bruner, J. S. y Ross, G. (1976). The role of tutoring in problem solving. Journal of Child Psychology \& Psychiatry \& Allied Disciplines. 17(2), 89-100. https://doi.org/bj3656 